\title{
Temperature Dependence of Kinetics for Reactive Diffusion in a Hypothetical Binary System
}

\author{
Masanori Kajihara* \\ Department of Materials Science and Engineering, Tokyo Institute of Technology, Yokohama 226-8502, Japan
}

A hypothetical binary system consisting of two primary solid solution phases $(\alpha$ and $\gamma)$ and one compound phase $(\beta)$ was considered in order to analyze theoretically the temperature dependence of kinetics for reactive diffusion. Assuming that migration of interface is controlled by volume diffusion in neighboring phases, the growth of the $\beta$ phase due to the reactive diffusion between the $\alpha$ and $\gamma$ phases in a semi-infinite diffusion couple was mathematically expressed as a function of the interdiffusion coefficients and the solubility ranges of the $\alpha, \beta$ and $\gamma$ phases. The assumption yields that the square of the thickness $l$ of the $\beta$ phase is proportional to the annealing time $t$ according to the parabolic relationship $l^{2}=K t$, where $K$ is the parabolic coefficient. The present attention was focused on the relationship between the temperature dependency of the growth rate and those of the interdiffusion coefficients, and hence the solubility ranges were assumed to be constant independently of the temperature and to take the same value for all the phases. On the contrary, the interdiffusion coefficient $D^{\theta}(\theta=\alpha, \beta, \gamma)$ was expressed as a function of the temperature $T$ by an Arrhenius equation of $D^{\theta}=D_{0}^{\theta} \exp \left(-Q^{\theta} / R T\right)$. For simplicity, however, $D_{0}^{\alpha}, D_{0}^{\beta}$ and $D_{0}^{\gamma}$ were considered equivalent. The temperature dependence of the parabolic coefficient $K$ was described also by an Arrhenius equation of $K=K_{0} \exp \left(-Q_{K} / R T\right)$, and then $K_{0}$ and $Q_{K}$ were evaluated for various combinations of $Q^{\alpha}, Q^{\beta}$ and $Q^{\gamma}$. The evaluation yields that $Q_{K}$ is equal to $Q^{\beta}$ at $Q^{\alpha}=Q^{\beta}=Q^{\gamma}$ and close to $Q^{\beta}$ at $Q^{\beta} \leq Q^{\alpha}$ and $Q^{\beta} \leq Q^{\gamma}$. Under such conditions, the temperature dependence of $D^{\beta}$ is estimated directly from that of $K$. On the other hand, $Q_{K}$ is greater than $Q^{\beta}$ at $Q^{\beta}>Q^{\alpha}$ or $Q^{\beta}>Q^{\gamma}$. In this case, such estimation becomes invalid.

(Received July 25, 2005; Accepted August 26, 2005; Published October 15, 2005)

Keywords: intermetallic compounds, bulk diffusion, analytical methods, reactive diffusion, kinetics

\section{Introduction}

There exist many binary alloy systems where intermetallic compounds appear as stable phases. ${ }^{1)}$ If a diffusion couple is prepared from two different pure metals in such a binary system and then annealed at an appropriate temperature $T$, some of the compounds may be recognized to form as layers at the interface between the two metals after certain periods due to reactive diffusion. Kinetics of the reactive diffusion was experimentally studied by many investigators for various alloy systems. ${ }^{2-20)}$ When the reactive diffusion is controlled by volume diffusion, the total thickness $l$ of the compound layers is described as a function of the annealing time $t$ by the parabolic relationship $l^{2}=K t$. Here, $K$ is the parabolic coefficient. The temperature dependence of $K$ may be expressed by an Arrhenius equation of $K=K_{0} \exp \left(-Q_{K} / R T\right)$, where $R$ is the gas constant. From experimental values of $K$ at different annealing temperatures, the pre-exponential factor $K_{0}$ and the activation enthalpy $Q_{K}$ can be determined by the least-squares method. Most of the experimental studies indicate that the temperature dependence of $K$ is reasonably described by the Arrhenius equation within experimental uncertainty. $^{2-20)}$ Thus, it is expected that the Arrhenius equation of $K$ derives representative properties of the interdiffusion in the diffusion couple. However, $K_{0}$ and $Q_{K}$ contain complex information of the temperature dependencies of the diffusion coefficients and the solubility ranges of the relevant phases. Consequently, such derivation is not so straightforward.

Recently, the reactive diffusion controlled by volume diffusion was theoretically analyzed by the present author using a mathematical model. ${ }^{21)}$ In the theoretical analysis, a hypothetical binary alloy system composed of two primary solid solution phases and one intermetallic compound was

*E-mail: kajihara@materia.titech.ac.jp considered, and then the growth rate of the compound was evaluated for various semi-infinite diffusion couples initially consisting of the two primary solid solution phases. The mathematical model was also used to analyze numerically the relationship between the temperature dependence of the interdiffusion in each phase and the kinetics of the reactive diffusion. ${ }^{22)}$ In the numerical analysis, the interdiffusion coefficient $D$ was described as a function of the annealing temperature $T$ by an Arrhenius equation of $D=$ $D_{0} \exp (-Q / R T)$, and the following assumptions were adopted: (a) the molar volume, the solubility range and the preexponential factor $D_{0}$ are constant and equivalent for all the phases; and (b) the activation enthalpy $Q$ is equivalent for the solution phases but different between the solution phase and the compound. The numerical analysis indicates that the equation $K=K_{0} \exp \left(-Q_{K} / R T\right)$ is reliable enough to express the temperature dependence of experimental values of $K$ but not necessarily completely exact. If $Q$ is smaller for the compound than for the solution phases, $Q_{K}$ is nearly equal to $Q$ of the compound. In such a case, the temperature dependence of $K$ corresponds well with that of $D$ of the compound. This relationship cannot hold good any longer, if $Q$ is greater for the compound than for the solution phases. In order to examine whether such conclusions are universally valid, assumption (b) was eliminated for numerical analysis in a previous study. ${ }^{23)}$ However, only limited combinations of $Q$ were treated between the two solution phases, and thus the validity could not be confirmed conclusively. In the present study, similar numerical analysis has been carried out extensively for various combinations of $Q$ among all the phases. Nevertheless, attention is still focused on the relationship between the temperature dependency of the kinetics and those of the interdiffusion coefficients of the constituent phases. Therefore, assumption (a) still remains for the numerical analysis in the present study. 


\section{Analysis}

A hypothetical binary A-B system composed of two primary solid solution phases and one intermetallic compound was adopted in previous studies. ${ }^{21-23)}$ The same binary system was treated also in the present study. The $\alpha$ and $\gamma$ phases are the primary solid solution phases of elements $\mathrm{A}$ and $\mathrm{B}$, respectively, and the $\beta$ phase is the compound. For the analysis, we consider a semi-infinite diffusion couple consisting of the $\alpha$ and $\gamma$ phases with initial compositions of $y^{\alpha 0}$ and $y^{\gamma 0}$, respectively. Here, $y$ is the mol fraction of element B. In the semi-infinite diffusion couple, the thickness is semi-infinite for the $\alpha$ and $\gamma$ phases, and the $\alpha / \gamma$ interface is flat. Therefore, the interdiffusion of elements $\mathrm{A}$ and $\mathrm{B}$ takes place unidirectionally along the direction perpendicular to the flat interface. This direction is hereafter called the diffusional direction. When the diffusion couple is annealed at temperature $T$ for an appropriate time, the $\beta$ phase will be produced as a layer at the interface due to the reactive diffusion between the $\alpha$ and $\gamma$ phases. The concentration profile of element $\mathrm{B}$ across the $\beta$ phase layer along the diffusional direction is schematically shown in Fig. 1. ${ }^{21)}$ In this figure, the ordinate indicates the mol fraction $y$, and the abscissa shows the distance $x$ measured from the initial position of the $\alpha / \gamma$ interface. Dashed lines and solid curves indicate the concentration profiles before and after annealing, respectively, and $z^{\alpha \beta}$ and $z^{\beta \gamma}$ show the positions of the $\alpha / \beta$ and $\beta / \gamma$ interfaces, respectively, after annealing. If the local equilibrium is established at each migrating interface during annealing, the compositions of the neighboring phases at the interface coincide with those of the corresponding phase boundaries at temperature $T$ in the phase diagram of the binary A-B system. As a result, the migration of the interface is controlled by the volume diffusion in the neighboring phases. In Fig. 1, $y^{\alpha \beta}$ and $y^{\beta \alpha}$ are the compositions of the $\alpha$ and $\beta$ phases, respectively, at the $\alpha / \beta$ interface, and $y^{\beta \gamma}$ and

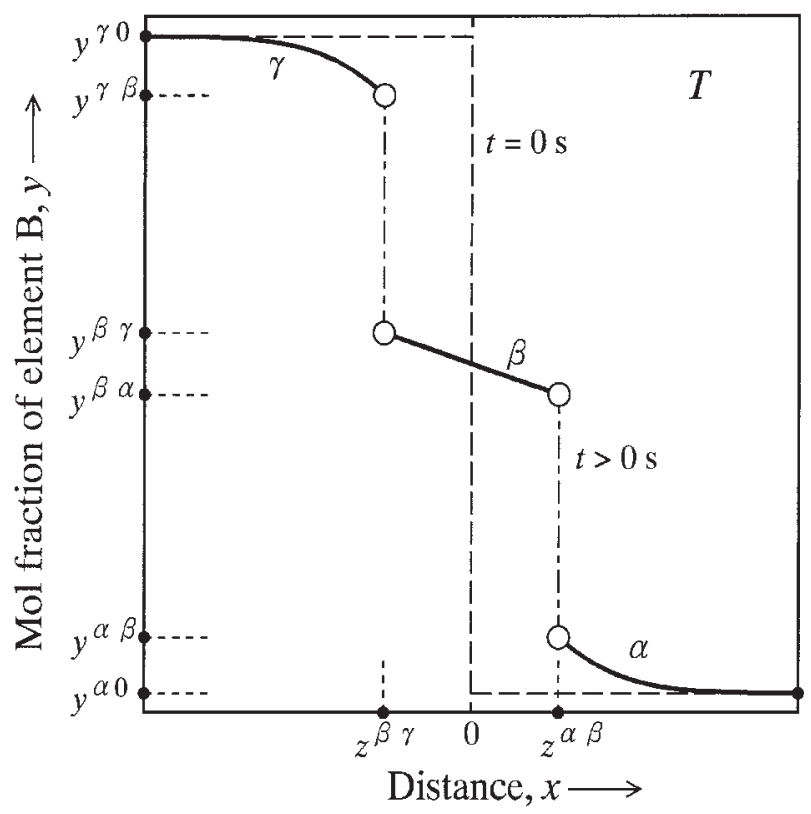

Fig. 1 Concentration profile of element $\mathrm{B}$ across the $\beta$ phase along the diffusional direction in the semi-infinite diffusion couple. ${ }^{21)}$ $y^{\gamma \beta}$ are those of the $\beta$ and $\gamma$ phases, respectively, at the $\beta / \gamma$ interface. The compositions $y^{\alpha \beta}, y^{\beta \alpha}, y^{\beta \gamma}$ and $y^{\gamma \beta}$ provide the boundary conditions, and those $y^{\alpha 0}$ and $y^{\gamma 0}$ give the initial conditions.

If the reactive diffusion is controlled by the volume diffusion, the positions $z^{\alpha \beta}$ and $z^{\beta \gamma}$ of the $\alpha / \beta$ and $\beta / \gamma$ interfaces are expressed as functions of the annealing time $t$ by the equations

$$
z^{\alpha \beta}=K^{\alpha \beta} \sqrt{4 D^{\alpha} t}=K^{\beta \alpha} \sqrt{4 D^{\beta} t}
$$

and

$$
z^{\beta \gamma}=K^{\beta \gamma} \sqrt{4 D^{\beta} t}=K^{\gamma \beta} \sqrt{4 D^{\gamma} t},
$$

respectively. ${ }^{24)}$ Here, $D^{\alpha}, D^{\beta}$ and $D^{\gamma}$ are the interdiffusion coefficients for volume diffusion in the $\alpha, \beta$ and $\gamma$ phases, respectively, and $K^{\alpha \beta}, K^{\beta \alpha}, K^{\beta \gamma}$ and $K^{\gamma \beta}$ are dimensionless coefficients. The thickness $l$ of the $\beta$ phase layer is readily obtained as the difference between $z^{\alpha \beta}$ and $z^{\beta \gamma}$, and hence the following equation is deduced from eq. (1) to express $l$ as a function of $t$.

$$
l^{2}=\left(z^{\alpha \beta}-z^{\beta \gamma}\right)^{2}=4 D^{\beta}\left(K^{\beta \alpha}-K^{\beta \gamma}\right)^{2} t=K t
$$

Here, $K$ is the parabolic coefficient defined as

$$
K \equiv 4 D^{\beta}\left(K^{\beta \alpha}-K^{\beta \gamma}\right)^{2} .
$$

The dimensionless coefficients are related to the initial and boundary conditions as follows:

$$
\begin{aligned}
c^{\beta \alpha}-c^{\alpha \beta}= & \frac{c^{\alpha 0}-c^{\alpha \beta}}{K^{\alpha \beta} \sqrt{\pi}\left\{1-\operatorname{erf}\left(K^{\alpha \beta}\right)\right\}} \exp \left\{-\left(K^{\alpha \beta}\right)^{2}\right\} \\
& +\frac{c^{\beta \gamma}-c^{\beta \alpha}}{K^{\beta \alpha} \sqrt{\pi}\left\{\operatorname{erf}\left(K^{\beta \alpha}\right)-\operatorname{erf}\left(K^{\beta \gamma}\right)\right\}} \\
& \times \exp \left\{-\left(K^{\beta \alpha}\right)^{2}\right\}
\end{aligned}
$$

and

$$
\begin{aligned}
c^{\gamma \beta}-c^{\beta \gamma}= & \frac{c^{\beta \alpha}-c^{\beta \gamma}}{K^{\beta \gamma} \sqrt{\pi}\left\{\operatorname{erf}\left(K^{\beta \alpha}\right)-\operatorname{erf}\left(K^{\beta \gamma}\right)\right\}} \exp \left\{-\left(K^{\beta \gamma}\right)^{2}\right\} \\
& +\frac{c^{\gamma 0}-c^{\gamma \beta}}{K^{\gamma \beta} \sqrt{\pi}\left\{1+\operatorname{erf}\left(K^{\gamma \beta}\right)\right\}} \exp \left\{-\left(K^{\gamma \beta}\right)^{2}\right\}
\end{aligned}
$$

Here, $c$ is the concentration of element B measured in mol per unit volume. The initial and boundary conditions are shown with the concentration $c$ in eq. (4), but indicated with the mol fraction $y$ in Fig. 1. However, $y$ is readily converted into $c$ by the equation $c=y / V_{\mathrm{m}}$, where $V_{\mathrm{m}}$ is the molar volume of the relevant phase. The following relationships are obtained from eq. (1):

$$
K^{\alpha \beta}=K^{\beta \alpha} \sqrt{D^{\beta} / D^{\alpha}}
$$

and

$$
K^{\gamma \beta}=K^{\beta \gamma} \sqrt{D^{\beta} / D^{\gamma}}
$$

Equation (5) shows that only two of the four dimensionless coefficients are independent. In the present analysis, $K^{\beta \alpha}$ and $K^{\beta \gamma}$ are chosen as the independent variables. Insertion of eq. (5) into eq. (4) yields two independent equations. Consequently, the two independent variables are finally determined from the two independent equations. 


\section{Results and Discussion}

Equations (2) to (5) indicate that the growth rate of the compound layer is controlled by the solubility ranges and the interdiffusion coefficients of the constituent phases in the diffusion couple. Hence, there are many parameters to determine the growth rate. As already mentioned in Section 1 , however, the attention is focused on the relationship between the temperature dependency of the growth rate and those of the interdiffusion coefficients. Thus, in the present analysis, the solubility ranges of all the phases are considered constant independently of the temperature. Furthermore, it is assumed that the molar volume $V_{\mathrm{m}}$ is independent of the composition and equivalent for all the phases. According to this assumption, the concentrations $c^{\alpha 0}, c^{\alpha \beta}, c^{\beta \alpha}, c^{\beta \gamma}, c^{\gamma \beta}$ and $c^{\gamma 0}$ in eq. (4) are automatically replaced with the mol fractions $y^{\alpha 0}, y^{\alpha \beta}, y^{\beta \alpha}, y^{\beta \gamma}, y^{\gamma \beta}$ and $y^{\gamma 0}$, respectively. The following initial and boundary conditions are adopted in the present analysis: $y^{\alpha 0}=0, y^{\alpha \beta}=0.1, y^{\beta \alpha}=0.45, y^{\beta \gamma}=0.55$, $y^{\gamma \beta}=0.9$ and $y^{\gamma 0}=1$. Hence, independently of the temperature, the solubility range $\Delta y$ is equal to 0.1 for all the phases, and the mean composition $y^{\beta}$ of the compound is identical to 0.5: $\Delta y=y^{\alpha \beta}-y^{\alpha 0}=y^{\beta \gamma}-y^{\beta \alpha}=y^{\gamma 0}-y^{\gamma \beta}=0.1 ; \quad$ and $y^{\beta}=\left(y^{\beta \alpha}+y^{\beta \gamma}\right) / 2=0.5$. The temperature dependence of the interdiffusion coefficient $D$ is described by the equation $D=D_{0} \exp (-Q / R T)$. Here, $D_{0}$ is the pre-exponential factor, and $Q$ is the activation enthalpy. The values of $Q^{\alpha}, Q^{\beta}$ and $Q^{\gamma}$ are varied from 50 to $100 \mathrm{~kJ} / \mathrm{mol}$, whereas the same value of $D_{0}=10^{-4} \mathrm{~m}^{2} / \mathrm{s}$ is used for all the phases in order to simplify the analysis. Using these parameters, the parabolic coefficient $K$ was numerically calculated as a function of $T$ from eqs. (3) $-(5)$ at a range of $T=700-1000 \mathrm{~K}$. The temperature dependence of $K$ was expressed by the equation $K=K_{0} \exp \left(-Q_{K} / R T\right)$, and the pre-exponential factor $K_{0}$ and the activation enthalpy $Q_{K}$ were determined in a manner similar to previous studies. ${ }^{22,23)}$

On the basis of the determination, dependencies of $K_{0}$ on $Q^{\alpha}$ and $Q^{\gamma}$ were evaluated for various values of $Q^{\beta}$. The results are shown as solid curves with constant values of $Q^{\gamma}=50-100 \mathrm{~kJ} / \mathrm{mol}$ in Fig. 2. In this figure, the abscissa indicates $Q^{\alpha}$, and the ordinate shows the logarithm of $K_{0}$. Figures 2(a), (b), (c), (d), (e) and (f) indicate the evaluations for $Q^{\beta}=50,60,70,80,90$ and $100 \mathrm{~kJ} / \mathrm{mol}$, respectively. At $Q^{\beta}=50 \mathrm{~kJ} / \mathrm{mol}$ in Fig. 2(a), $K_{0}$ monotonically increases with increasing value of $Q^{\alpha}$ for each solid curve. For all the phases, the solubility range $\Delta y$ and the pre-exponential factor $D_{0}$ are identical to 0.1 and $10^{-4} \mathrm{~m}^{2} / \mathrm{s}$, respectively, as mentioned earlier. Furthermore, the mean composition $y^{\beta}$ of the compound is equal to 0.5 . Thus, the effects of $Q^{\alpha}$ and $Q^{\gamma}$ on the temperature dependence of $K$ are equivalent each other. As a result, $K_{0}$ monotonically increases also with increasing value of $Q^{\gamma}$ at a constant value of $Q^{\alpha}$. On the other hand, at $Q^{\beta}=60 \mathrm{~kJ} / \mathrm{mol}$ in Fig. 2(b), $K_{0}$ slightly decreases with increasing value of $Q^{\alpha}$, and reaches the minimum value at $Q^{\alpha} \cong 60 \mathrm{~kJ} / \mathrm{mol}$. After taking the minimum value, $K_{0}$ gradually increases with increasing value of $Q^{\alpha}$. Due to the equivalence between the effects of $Q^{\alpha}$ and $Q^{\gamma}$ on the temperature dependence of $K$, the appearance of the minimum point is also the reason why $K_{0}$ is smaller for the solid curves with $Q^{\gamma}=60$ and $70 \mathrm{~kJ} / \mathrm{mol}$ than for those with $Q^{\gamma}=$
50 and $80 \mathrm{~kJ} / \mathrm{mol}$ but greater for those with $Q^{\gamma}=90$ and $100 \mathrm{~kJ} / \mathrm{mol}$ than for those with $Q^{\gamma}=50$ and $80 \mathrm{~kJ} / \mathrm{mol}$. At $Q^{\beta}=70 \mathrm{~kJ} / \mathrm{mol}$ in Fig. 2(c), however, the minimum point shifts to $Q^{\alpha} \cong 70 \mathrm{~kJ} / \mathrm{mol}$ for the solid curves with $Q^{\gamma}=$ $60-100 \mathrm{~kJ} / \mathrm{mol}$, but almost stays at $Q^{\alpha} \cong 60 \mathrm{~kJ} / \mathrm{mol}$ for that with $Q^{\gamma}=50 \mathrm{~kJ} / \mathrm{mol}$. On the contrary, at $Q^{\beta}=80 \mathrm{~kJ} / \mathrm{mol}$ in Fig. 2(d), the minimum point nearly remains at $Q^{\alpha} \cong 60$ and $70 \mathrm{~kJ} / \mathrm{mol}$ for the solid curves with $Q^{\gamma}=50$ and $60 \mathrm{~kJ} / \mathrm{mol}$, respectively, but relocates to $Q^{\alpha} \cong 80 \mathrm{~kJ} / \mathrm{mol}$ for those with $Q^{\gamma}=70-100 \mathrm{~kJ} / \mathrm{mol}$. In the case of $Q^{\beta}=100 \mathrm{~kJ} / \mathrm{mol}, K_{0}$ increases with increasing value of $Q^{\gamma}$ at $Q^{\alpha}=50 \mathrm{~kJ} / \mathrm{mol}$, but decreases with increasing value of $Q^{\gamma}$ at $Q^{\alpha}=100 \mathrm{~kJ} / \mathrm{mol}$. Consequently, $K_{0}$ varies depending on $Q^{\alpha}$ and $Q^{\gamma}$ in a complicated manner as shown in Fig. 2(f).

For various values of $Q^{\beta}$, dependencies of $Q_{K}$ on $Q^{\alpha}$ and $Q^{\gamma}$ were evaluated together with those of $K_{0}$ on $Q^{\alpha}$ and $Q^{\gamma}$. The results are indicated as solid curves with constant values of $Q^{\gamma}=50-100 \mathrm{~kJ} / \mathrm{mol}$ in Fig. 3. The evaluations for $Q^{\beta}=50,60,70,80,90$ and $100 \mathrm{~kJ} / \mathrm{mol}$ are shown in Figs. 3(a), (b), (c), (d), (e) and (f), respectively. Figures 3(a) and (f) represent the results obtained in a previous study. ${ }^{23)}$ At $Q^{\beta}=50 \mathrm{~kJ} / \mathrm{mol}$ in Fig. 3(a), $Q_{K}$ lightly decreases with increasing value of $Q^{\alpha}$, and attains the minimum value at $Q^{\alpha} \cong 60 \mathrm{~kJ} / \mathrm{mol}$. After attaining the minimum value, $Q_{K}$ gradually increases with increasing value of $Q^{\alpha}$. However, such variations of $Q_{K}$ are rather small, and thus $Q_{K}$ is close to $Q^{\beta}$. On the other hand, at $Q^{\beta}=60 \mathrm{~kJ} / \mathrm{mol}$ in Fig. 3(b), the minimum point migrates to $Q^{\alpha} \cong 70 \mathrm{~kJ} / \mathrm{mol}$ for all the solid curves. $Q_{K}$ is close to $Q^{\beta}$ at $Q^{\alpha}=60-100 \mathrm{~kJ} / \mathrm{mol}$ and $Q^{\gamma}=60-100 \mathrm{~kJ} / \mathrm{mol}$, but slightly greater than $Q^{\beta}$ at $Q^{\alpha}=50 \mathrm{~kJ} / \mathrm{mol}$ or $Q^{\gamma}=50 \mathrm{~kJ} / \mathrm{mol}$. It is interesting to note that the minimum point of the solid curve appears at different values of $Q^{\alpha}$ for $K_{0}$ and $Q_{K}$ as indicated in Figs. 2(b) and 3(b). According to the results in Figs. 3(c)-(f), $Q_{K}$ is close to $Q^{\beta}$ at $Q^{\alpha}=70-100 \mathrm{~kJ} / \mathrm{mol}$ and $Q^{\gamma}=70-100 \mathrm{~kJ} / \mathrm{mol}$ for $Q^{\beta}=70 \mathrm{~kJ} / \mathrm{mol}$, at $Q^{\alpha}=80-100 \mathrm{~kJ} / \mathrm{mol}$ and $Q^{\gamma}=80-100$ $\mathrm{kJ} / \mathrm{mol}$ for $Q^{\beta}=80 \mathrm{~kJ} / \mathrm{mol}$, at $Q^{\alpha}=90-100 \mathrm{~kJ} / \mathrm{mol}$ and $Q^{\gamma}=90-100 \mathrm{~kJ} / \mathrm{mol}$ for $Q^{\beta}=90 \mathrm{~kJ} / \mathrm{mol}$, and at $Q^{\alpha}=100$ $\mathrm{kJ} / \mathrm{mol}$ and $Q^{\gamma}=100 \mathrm{~kJ} / \mathrm{mol}$ for $Q^{\beta}=100 \mathrm{~kJ} / \mathrm{mol}$. However, $Q_{K}$ is greater than $Q^{\beta}$ at $Q^{\alpha}=50-60 \mathrm{~kJ} / \mathrm{mol}$ or $Q^{\gamma}=$ $50-60 \mathrm{~kJ} / \mathrm{mol}$ for $Q^{\beta}=70 \mathrm{~kJ} / \mathrm{mol}$, at $Q^{\alpha}=50-70 \mathrm{~kJ} / \mathrm{mol}$ or $Q^{\gamma}=50-70 \mathrm{~kJ} / \mathrm{mol}$ for $Q^{\beta}=80 \mathrm{~kJ} / \mathrm{mol}$, at $Q^{\alpha}=50-80 \mathrm{~kJ} /$ mol or $Q^{\gamma}=50-80 \mathrm{~kJ} / \mathrm{mol}$ for $Q^{\beta}=90 \mathrm{~kJ} / \mathrm{mol}$, and at $Q^{\alpha}=50-90 \mathrm{~kJ} / \mathrm{mol}$ or $Q^{\gamma}=50-90 \mathrm{~kJ} / \mathrm{mol}$ for $Q^{\beta}=100$ $\mathrm{kJ} / \mathrm{mol}$. Thus, it is concluded that $Q_{K}$ is close to $Q^{\beta}$ at $Q^{\alpha} \geq$ $Q^{\beta}$ and $Q^{\gamma} \geq Q^{\beta}$ but greater than $Q^{\beta}$ at $Q^{\alpha}<Q^{\beta}$ or $Q^{\gamma}<Q^{\bar{\beta}}$.

The results in Fig. 3 are shown in a different manner in Fig. 4. In this figure, solid curves indicate the evaluations with constant values of $Q^{\beta}=50-100 \mathrm{~kJ} / \mathrm{mol}$. Open circles show values of $Q_{K}$ at $Q^{\alpha}=Q^{\beta}$, and a dashed straight line indicates a relationship of $Q_{K}=Q^{\alpha}$. Figures 4(a), (b), (c), (d), (e) and (f) show the results for $Q^{\gamma}=50,60,70,80,90$ and $100 \mathrm{~kJ} / \mathrm{mol}$, respectively. The results in Figs. 4(a) and (f) were reported in a previous study. ${ }^{23)}$ At $Q^{\gamma}=50 \mathrm{~kJ} / \mathrm{mol}$ in Fig. 4(a), $Q_{K}$ is rather insensitive to the variation of $Q^{\alpha}$, and close to $Q^{\beta}$ for the solid curve with $Q^{\beta}=50 \mathrm{~kJ} / \mathrm{mol}$. The open circle of this solid curve lies on the dashed line. However, $Q_{K}$ is greater than $Q^{\beta}$ for the solid curves with $Q^{\beta}=60-100 \mathrm{~kJ} / \mathrm{mol}$. Thus, the open circles of these solid curves are distributed on the upper-side of the dashed line. On 

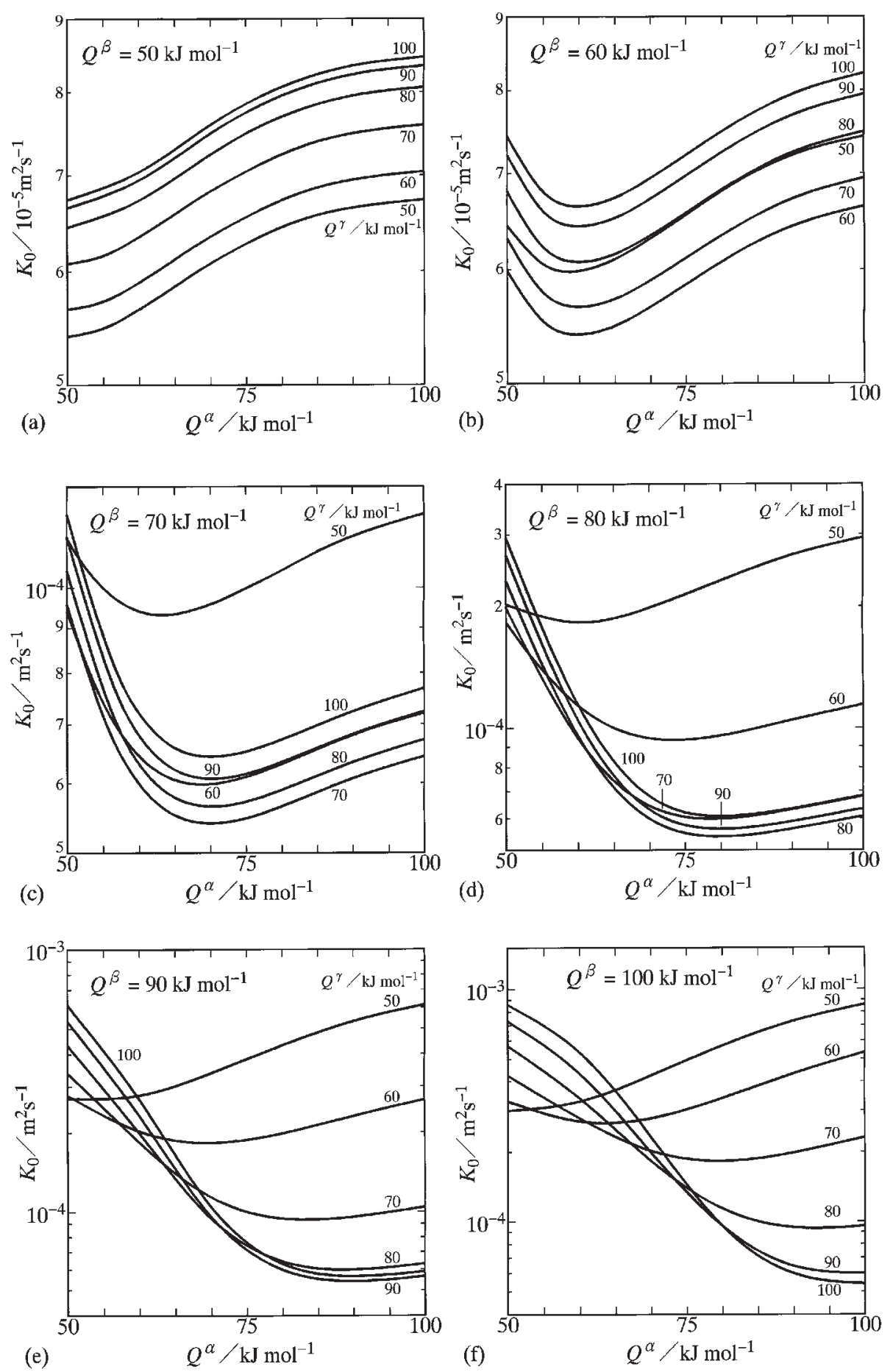

Fig. 2 Dependencies of $K_{0}$ on $Q^{\alpha}$ and $Q^{\gamma}$ for (a) $Q^{\beta}=50 \mathrm{~kJ} / \mathrm{mol}$, (b) $Q^{\beta}=60 \mathrm{~kJ} / \mathrm{mol}$, (c) $Q^{\beta}=70 \mathrm{~kJ} / \mathrm{mol}$, (d) $Q^{\beta}=80 \mathrm{~kJ} / \mathrm{mol}$, (e) $Q^{\beta}=90 \mathrm{~kJ} / \mathrm{mol}$ and (f) $Q^{\beta}=100 \mathrm{~kJ} / \mathrm{mol}$.

the other hand, at $Q^{\gamma}=60 \mathrm{~kJ} / \mathrm{mol}$ in Fig. $4(\mathrm{~b}), Q_{K}$ is close to $Q^{\beta}$ at $Q^{\alpha} \geq 50 \mathrm{~kJ} / \mathrm{mol}$ for the solid curve with $Q^{\beta}=50 \mathrm{~kJ} /$ mol and at $Q^{\alpha} \geq 60 \mathrm{~kJ} / \mathrm{mol}$ for that with $Q^{\beta}=60 \mathrm{~kJ} / \mathrm{mol}$, but slightly greater than $Q^{\beta}$ at $Q^{\alpha}<60 \mathrm{~kJ} / \mathrm{mol}$ for the latter solid curve. The open circle of the solid curve with $Q^{\beta}=$ $60 \mathrm{~kJ} / \mathrm{mol}$ is completely located on the dashed line, but that of the solid curve with $Q^{\beta}=50 \mathrm{~kJ} / \mathrm{mol}$ lies slightly below the dashed line. For the solid curves with $Q^{\beta}=70-100 \mathrm{~kJ} /$ mol, however, $Q_{K}$ is greater than $Q^{\beta}$. As can be seen in Figs. 4(c)-(f), $Q_{K}$ is nearly equal to $Q^{\beta}$ at $Q^{\alpha} \geq Q^{\beta}$ for $Q^{\beta}=50-70 \mathrm{~kJ} / \mathrm{mol}$ at $Q^{\gamma}=70 \mathrm{~kJ} / \mathrm{mol}$, for $Q^{\beta}=50-80 \mathrm{~kJ} /$ mol at $Q^{\gamma}=80 \mathrm{~kJ} / \mathrm{mol}$, for $Q^{\beta}=50-90 \mathrm{~kJ} / \mathrm{mol}$ at $Q^{\gamma}=90$ $\mathrm{kJ} / \mathrm{mol}$ and for $Q^{\beta}=50-100 \mathrm{~kJ} / \mathrm{mol}$ at $Q^{\gamma}=100 \mathrm{~kJ} / \mathrm{mol}$. For such combinations of $Q^{\beta}$ and $Q^{\gamma}$, however, $Q_{K}$ is greater than $Q^{\beta}$ at $Q^{\alpha}<Q^{\beta}$. On the contrary, independently of $Q^{\alpha}$, $Q_{K}$ is greater than $Q^{\beta}$ for $Q^{\beta}=80-100 \mathrm{~kJ} / \mathrm{mol}$ at $Q^{\gamma}=70$ $\mathrm{kJ} / \mathrm{mol}$, for $Q^{\beta}=90-100 \mathrm{~kJ} / \mathrm{mol}$ at $Q^{\gamma}=80 \mathrm{~kJ} / \mathrm{mol}$ and for $Q^{\beta}=100 \mathrm{~kJ} / \mathrm{mol}$ at $Q^{\gamma}=90 \mathrm{~kJ} / \mathrm{mol}$. These relationships indicate that $Q_{K}$ is close to $Q^{\beta}$ at $Q^{\alpha} \geq Q^{\beta}$ for $Q^{\beta} \leq Q^{\gamma}$. If such conditions are not realized, $Q_{K}$ becomes greater than $Q^{\beta}$.

From the evaluations in Fig. 3, the dependence of $Q_{K}$ 

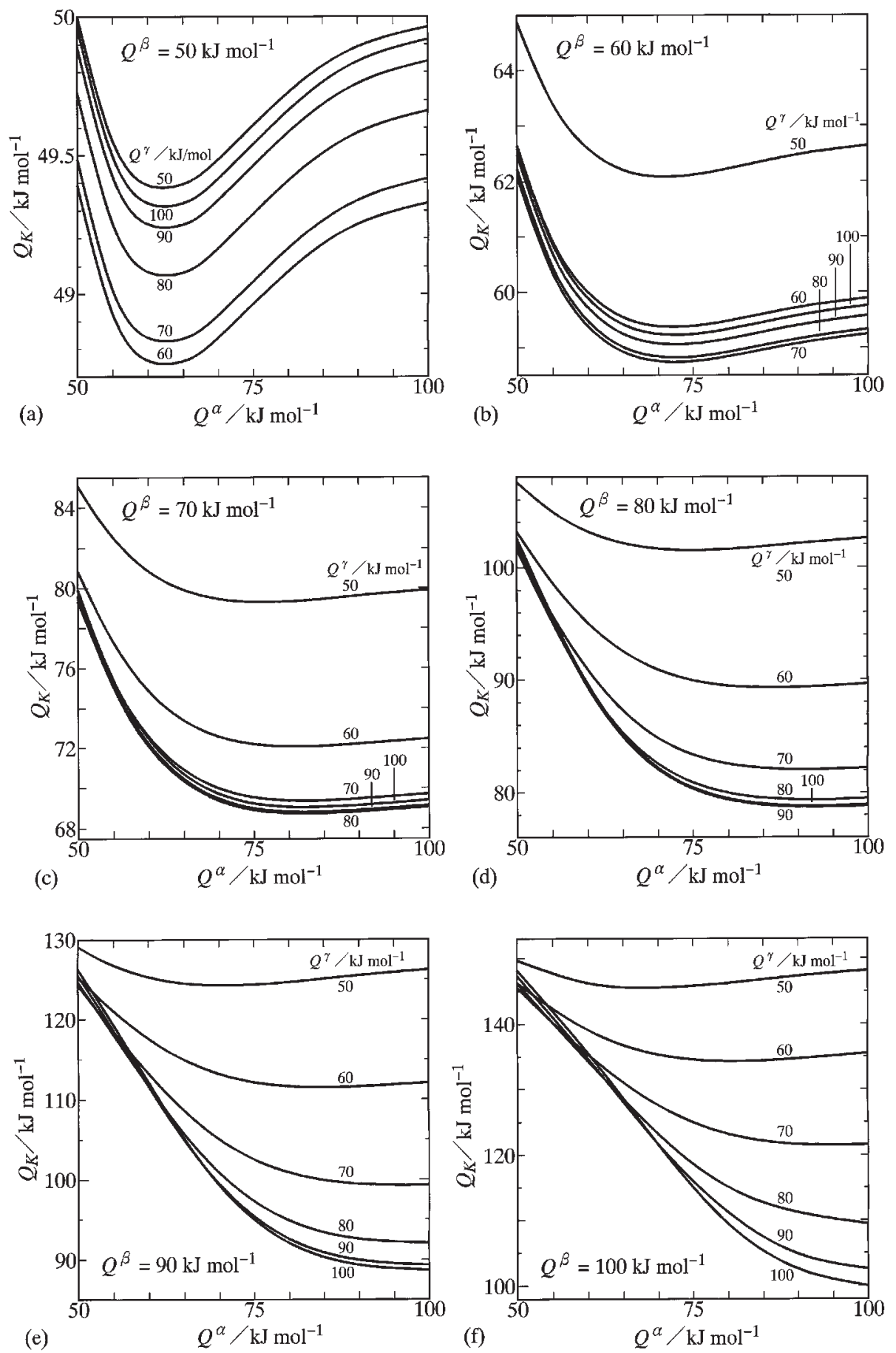

Fig. 3 Dependencies of $Q_{K}$ on $Q^{\alpha}$ and $Q^{\gamma}$ for (a) $Q^{\beta}=50 \mathrm{~kJ} / \mathrm{mol}$, (b) $Q^{\beta}=60 \mathrm{~kJ} / \mathrm{mol}$, (c) $Q^{\beta}=70 \mathrm{~kJ} / \mathrm{mol}$, (d) $Q^{\beta}=80 \mathrm{~kJ} / \mathrm{mol}$, (e) $Q^{\beta}=90 \mathrm{~kJ} / \mathrm{mol}$ and (f) $Q^{\beta}=100 \mathrm{~kJ} / \mathrm{mol}$.

on $Q^{\beta}$ is illustrated for various values of $Q^{\alpha}$ and $Q^{\gamma}$. The results with constant values of $Q^{\gamma}=50-100 \mathrm{~kJ} / \mathrm{mol}$ are shown as solid curves in Fig. 5. In this figure, the abscissa indicates $Q^{\beta}$, and the ordinate shows the ratio of $Q_{K}$ to $Q^{\beta}$. Open circles indicate values of the ratio $Q_{K} / Q^{\beta}$ at $Q^{\beta}=Q^{\gamma}$, and a horizontal dashed line shows a value of $Q_{K} / Q^{\beta}=1$. The evaluations for $Q^{\alpha}=50,60,70,80,90$ and $100 \mathrm{~kJ} / \mathrm{mol}$ are indicated in Figs. 5(a), (b), (c), (d), (e) and (f), respectively. At $Q^{\alpha}=50 \mathrm{~kJ} / \mathrm{mol}$ in Fig. $5(\mathrm{a})$, the ratio $Q_{K} / Q^{\beta}$ is equal to unity at $Q^{\beta}=50 \mathrm{~kJ} / \mathrm{mol}$ for the solid curve with $Q^{\gamma}=50 \mathrm{~kJ} / \mathrm{mol}$, and close to unity at $Q^{\beta}=$
$50 \mathrm{~kJ} / \mathrm{mol}$ for those with $Q^{\gamma}=60-100 \mathrm{~kJ} / \mathrm{mol}$. As $Q^{\beta}$ increases, the ratio $Q_{K} / Q^{\beta}$ monotonically increases, and becomes greater for $Q^{\gamma}=50 \mathrm{~kJ} / \mathrm{mol}$ than for $Q^{\gamma}=$ $60-100 \mathrm{~kJ} / \mathrm{mol}$. On the other hand, at $Q^{\alpha}=60 \mathrm{~kJ} / \mathrm{mol}$ in Fig. 5(b), the ratio $Q_{K} / Q^{\beta}$ is equal to unity at $Q^{\beta}=60 \mathrm{~kJ} /$ mol for $Q^{\gamma}=60 \mathrm{~kJ} / \mathrm{mol}$, and close to unity at $Q^{\beta} \leq 60 \mathrm{~kJ} /$ mol for $Q^{\gamma}=70-100 \mathrm{~kJ} / \mathrm{mol}$. Although the ratio $Q_{K} / Q^{\beta}$ for $Q^{\gamma}=60 \mathrm{~kJ} / \mathrm{mol}$ is still smaller than that for $Q^{\gamma}=50 \mathrm{~kJ} / \mathrm{mol}$, it becomes greater than that for $Q^{\gamma}=70-100 \mathrm{~kJ} / \mathrm{mol}$ at $Q^{\beta}>60 \mathrm{~kJ} / \mathrm{mol}$. At $Q^{\alpha}=70 \mathrm{~kJ} / \mathrm{mol}$ in Fig. 5(c), the ratio $Q_{K} / Q^{\beta}$ is equal to unity at $Q^{\beta}=70$ for $Q^{\gamma}=70 \mathrm{~kJ} / \mathrm{mol}$, and 

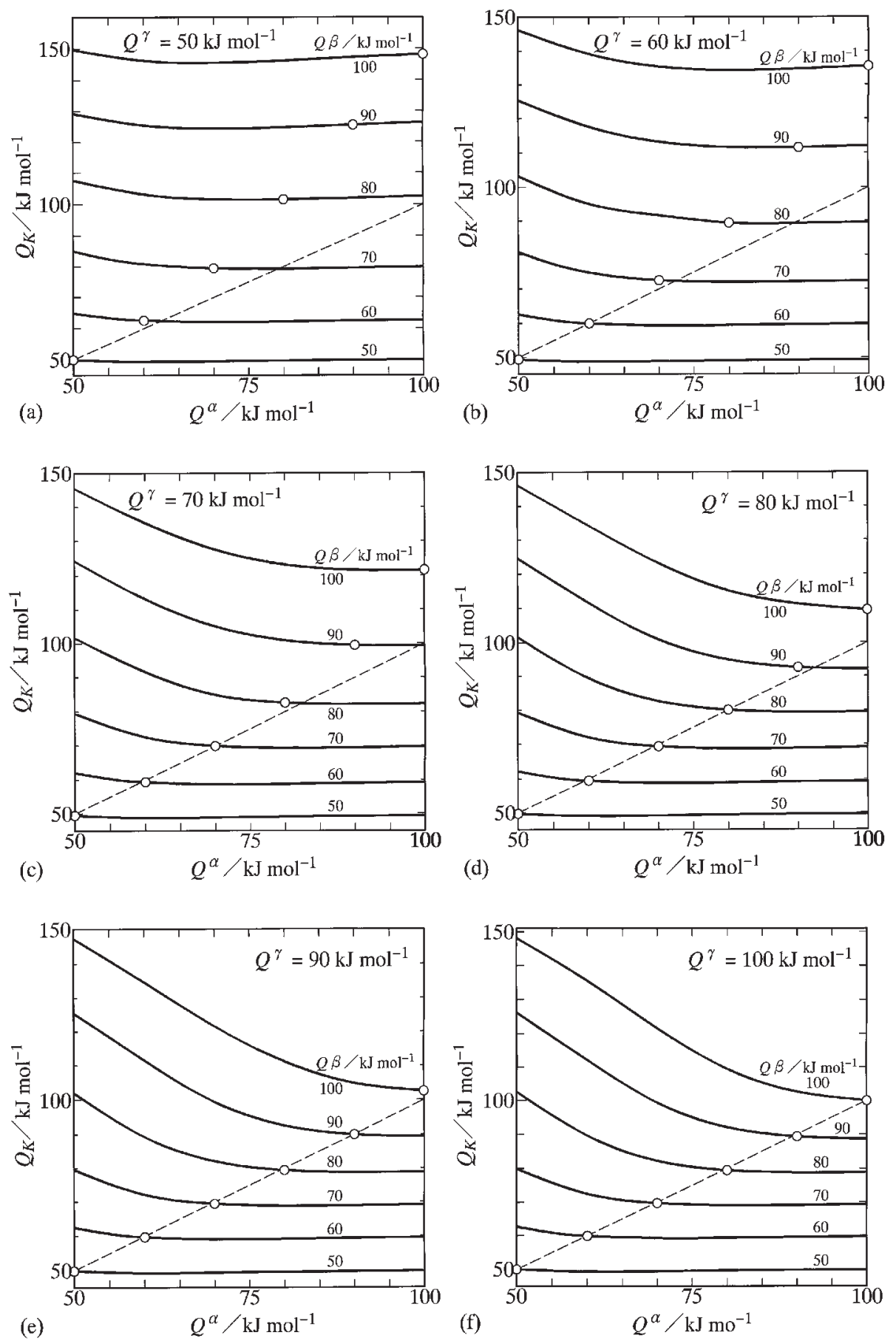

Fig. 4 Dependencies of $Q_{K}$ on $Q^{\alpha}$ and $Q^{\beta}$ for (a) $Q^{\gamma}=50 \mathrm{~kJ} / \mathrm{mol}$, (b) $Q^{\gamma}=60 \mathrm{~kJ} / \mathrm{mol}$, (c) $Q^{\gamma}=70 \mathrm{~kJ} / \mathrm{mol}$, (d) $Q^{\gamma}=80 \mathrm{~kJ} / \mathrm{mol}$, (e) $Q^{\gamma}=90 \mathrm{~kJ} / \mathrm{mol}$ and (f) $Q^{\gamma}=100 \mathrm{~kJ} / \mathrm{mol}$.

close to unity at $Q^{\beta}=50 \mathrm{~kJ} / \mathrm{mol}$ for $Q^{\gamma}=50 \mathrm{~kJ} / \mathrm{mol}$, at $Q^{\beta} \leq 60 \mathrm{~kJ} / \mathrm{mol}$ for $Q^{\gamma}=60 \mathrm{~kJ} / \mathrm{mol}$ and at $Q^{\beta} \leq 70 \mathrm{~kJ} / \mathrm{mol}$ for $Q^{\gamma}=70-100 \mathrm{~kJ} / \mathrm{mol}$. In the case of $Q^{\alpha}=80 \mathrm{~kJ} / \mathrm{mol}$ in Fig. 5(d), however, the ratio $Q_{K} / Q^{\beta}$ is equal to unity at $Q^{\beta}=$ 80 for $Q^{\gamma}=80 \mathrm{~kJ} / \mathrm{mol}$, and close to unity at $Q^{\beta} \leq 80 \mathrm{~kJ} / \mathrm{mol}$ for $Q^{\gamma}=90-100 \mathrm{~kJ} / \mathrm{mol}$ as well as at $Q^{\beta}=50 \mathrm{~kJ} / \mathrm{mol}$ for $Q^{\gamma}=50 \mathrm{~kJ} / \mathrm{mol}$, at $Q^{\beta} \leq 60 \mathrm{~kJ} / \mathrm{mol}$ for $Q^{\gamma}=60 \mathrm{~kJ} / \mathrm{mol}$ and at $Q^{\beta} \leq 70 \mathrm{~kJ} / \mathrm{mol}$ for $Q^{\gamma}=70 \mathrm{~kJ} / \mathrm{mol}$. Considering the results at $Q^{\alpha}=90$ and $100 \mathrm{~kJ} / \mathrm{mol}$ in Figs. 5(e) and (f), it is finally concluded that $Q_{K}$ is exactly equal to $Q^{\beta}$ at $Q^{\alpha}=$ $Q^{\beta}=Q^{\gamma}$ and close to $Q^{\beta}$ at $Q^{\beta} \leq Q^{\alpha}$ and $Q^{\beta} \leq Q^{\gamma}$. Under such conditions, the activation enthalpy of the interdiffusion coefficient in the growing compound layer is estimated directly from that of the parabolic coefficient. On the other hand, $Q_{K}$ is greater than $Q^{\beta}$ at $Q^{\beta}>Q^{\alpha}$ or $Q^{\beta}>Q^{\gamma}$. In this case, such estimation becomes invalid.

The reactive diffusion was experimentally studied for many binary systems consisting of elements with considerably different melting temperatures. ${ }^{10-20)}$ When the melting temperature is much higher for the $\alpha$ phase than for the $\beta$ and $\gamma$ phases, the interdiffusion occurs much slower in the former phase than in the latter phases at a constant annealing temperature. Furthermore, the stable crystal structure of a compound is usually an ordered lattice in many binary 

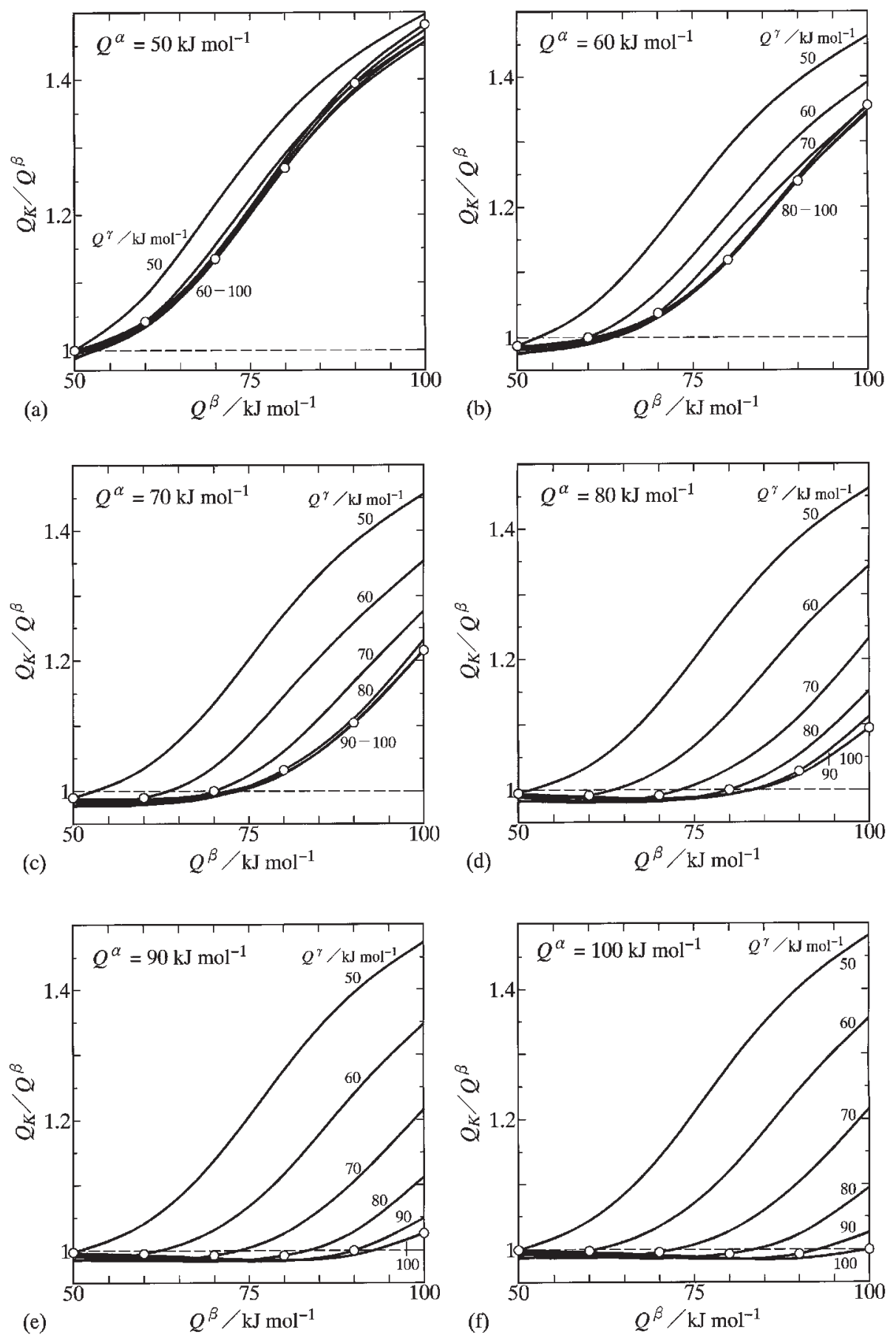

Fig. 5 Dependencies of the ratio $Q_{K} / Q^{\beta}$ on $Q^{\beta}$ and $Q^{\gamma}$ for (a) $Q^{\alpha}=50 \mathrm{~kJ} / \mathrm{mol}$, (b) $Q^{\alpha}=60 \mathrm{~kJ} / \mathrm{mol}$, (c) $Q^{\alpha}=70 \mathrm{~kJ} / \mathrm{mol}$, (d) $Q^{\alpha}=80 \mathrm{~kJ} /$ mol, (e) $Q^{\alpha}=90 \mathrm{~kJ} / \mathrm{mol}$ and (f) $Q^{\alpha}=100 \mathrm{~kJ} / \mathrm{mol}$.

systems. ${ }^{1)}$ Thus, the interdiffusion in the $\beta$ phase should be more sluggish than that in the $\gamma$ phase unless the melting temperature is much lower for the $\beta$ phase than for the $\gamma$ phase. Under such conditions, we may expect $Q^{\alpha}>$ $Q^{\beta}>Q^{\gamma}$. According to the conclusions mentioned above, the temperature dependence of the interdiffusion coefficient in the $\beta$ phase cannot be estimated directly from that of the parabolic coefficient at $Q^{\alpha}>Q^{\beta}>Q^{\gamma}$. In this case, experimental information on the interdiffusion is essentially important for reliable estimation of the activation enthalpy of the interdiffusion coefficient in the compound.

\section{Conclusions}

The temperature dependence of the kinetics of reactive diffusion was theoretically analyzed for the hypothetical binary system composed of one compound phase $(\beta)$ and two primary solid solution phases $(\alpha$ and $\gamma$ ). The growth rate of the $\beta$ phase during the reactive diffusion between the $\alpha$ and $\gamma$ phases in a semi-infinite diffusion couple was expressed as a function of the interdiffusion coefficients and the solubility ranges of the $\alpha, \beta$ and $\gamma$ phases using the mathematical model reported in a previous study. ${ }^{21}$ For simplicity, however, the solubility ranges of all the 
phases were assumed to be constant and equivalent. On the contrary, the interdiffusion coefficient $D^{\theta}$ was described as a function of the temperature $T$ by the equation $D^{\theta}=$ $D_{0}^{\theta} \exp \left(-Q^{\theta} / R T\right)$. Here, $D_{0}^{\theta}$ is the pre-exponential factor, $Q^{\theta}$ is the activation enthalpy, $R$ is the gas constant, and $\theta$ stands for $\alpha, \beta$ and $\gamma$. Furthermore, we assumed $D_{0}^{\alpha}=D_{0}^{\beta}=D_{0}^{\gamma}$. For the reactive diffusion controlled by the volume diffusion, the square of the thickness $l$ of the $\beta$ phase is proportional to the annealing time $t$ as $l^{2}=K t$. The temperature dependence of the parabolic coefficient $K$ was expressed by the equation of $K=K_{0} \exp \left(-Q_{K} / R T\right)$, and then the pre-exponential factor $K_{0}$ and the activation enthalpy $Q_{K}$ were evaluated for different combinations of $Q^{\alpha}, Q^{\beta}$ and $Q^{\gamma}$. In the case of $Q^{\alpha}=Q^{\beta}=Q^{\gamma}, Q_{K}$ is exactly equal to $Q^{\beta}$. $Q_{K}$ is still close to $Q^{\beta}$ at $Q^{\beta} \leq Q^{\alpha}$ and $Q^{\beta} \leq Q^{\gamma}$. Under such conditions, the temperature dependence of $D^{\beta}$ is estimated directly from that of $K$. Unfortunately, however, $Q_{K}$ becomes greater than $Q^{\beta}$ at $Q^{\beta}>Q^{\alpha}$ or $Q^{\beta}>Q^{\gamma}$. In such a case, $Q^{\beta}$ cannot be estimated directly from $Q_{K}$.

\section{Acknowledgements}

The present study was supported by a Grant-in-Aid for Scientific Research from the Ministry of Education, Culture, Sports, Science and Technology of Japan.

\section{REFERENCES}

1) T. B. Massalski, H. Okamoto, P. R. Subramanian and L. Kacprzak: Binary Alloy Phase Diagram (ASM International, Materials Park, $\mathrm{OH}, 1990)$ vol. 1-3.
2) B. Lustman and R. F. Mehl: Trans. Met. Soc. AIME 147 (1942) 369394.

3) D. Horstmann: Stahl Eisen 73 (1953) 659-665

4) S. Storchheim, J. L. Zambrow and H. H. Hausner: Trans. Met. Soc. AIME 200 (1954) 269-274.

5) G. V. Kidson and G. D. Miller: J. Nucl. Mater. 12 (1964) 61-69.

6) K. Shibata, S. Morozumi and S. Koda: J. Jpn. Inst. Met. 30 (1966) 382388.

7) K. Hirano and Y. Ipposhi: J. Jpn. Inst. Met. 32 (1968) 815-821.

8) M. M. P. Janssen: Metall. Trans. 4 (1973) 1623-1633.

9) G. F. Bastin and G. D. Rieck: Metall. Trans. 5 (1974) 1817-1826.

10) M. Onishi and H. Fujibuchi: Trans. JIM 16 (1975) 539-547.

11) EI-B. Hannech and C. R. Hall: Mater. Sci. Tech. 8 (1992) 817-824.

12) P. T. Vianco, P. F. Hlava and A. L. Kilgo: J. Electron. Mater. 23 (1994) 583-594.

13) M. Watanabe, Z. Horita and M. Nemoto: Interface Science 4 (1997) 229-241

14) S. Choi, T. R. Bieler, J. P. Lucas and K. N. Subramanian: J. Electron. Mater. 28 (1999) 1209-1215.

15) T. Yamada, K. Miura, M. Kajihara, N. Kurokawa and K. Sakamoto: Mater. Sci. Eng. A 390 (2005) 118-126.

16) T. Takenaka, S. Kano, M. Kajihara, N. Kurokawa and K. Sakamoto: Mater. Sci. Eng. A 396 (2005) 115-123.

17) K. Suzuki, S. Kano, M. Kajihara, N. Kurokawa and K. Sakamoto: Mater. Trans. 46 (2005) 969-973.

18) T. Takenaka, S. Kano, M. Kajihara, N. Kurokawa and K. Sakamoto: Mater. Trans. 46 (2005) 1825-1832.

19) M. Mita, M. Kajihara, N. Kurokawa and K. Sakamoto: Mater. Sci. Eng. A 403 (2005) 269-275.

20) T. Takenaka, M. Kajihara, N. Kurokawa and K. Sakamoto: Mater. Sci. Eng. A 406 (2005) 134-141.

21) M. Kajihara: Acta Mater. 52 (2004) 1193-1200.

22) M. Kajihara: Mater. Sci. Eng. A 403 (2005) 234-240.

23) M. Kajihara: Defect and Diffusion Forum, in press.

24) W. Jost: Diffusion of Solids, Liquids, Gases (Academic Press, New York, 1960) p. 68. 